\title{
Initiation of oblique detonation waves induced by a blunt wedge in stoichiometric hydrogen-air mixtures
}

\author{
Yishen Fang ${ }^{\mathrm{a}, \mathrm{b}}$, Zijian Zhang ${ }^{\mathrm{a}, \mathrm{b}}$, Zongmin $\mathrm{Hu}^{\mathrm{a}}{ }^{\mathrm{a}, *}$, Xi Deng ${ }^{\mathrm{c}}$ \\ a State Key Laboratory of High Temperature Gas Dynamics, Institute of Mechanics, Chinese Academy of Sciences, Beijing, 100190, China \\ b School of Engineering Sciences, University of Chinese Academy of Sciences, Beijing, 100049, China \\ c Tokyo Institute of Technology, Tokyo, 152-8550, Japan
}

\section{A R T I C L E I N F O}

\section{Article history:}

Received 21 January 2019

Received in revised form 13 May 2019

Accepted 14 June 2019

Available online 29 June 2019

\section{Keywords:}

Oblique detonation wave

Blunt wedge

Initiation

Hydrogen

\begin{abstract}
A B S T R A C T
Two-dimensional, oblique detonation waves (ODWs) in a stoichiometric hydrogen-air mixture are simulated with the reactive Euler equations using a detailed chemical reaction model. This study focuses on blunt wedge induced ODWs, which are not only influenced by inflow parameters but also the size of the blunt body. With the inflow parameters of flight altitude of $30 \mathrm{~km}$ and flight Mach number $M_{0}$ of $8-10$, the numerical results demonstrate that the blunt wedge is crucial to initiate the ODW. In the case of $M_{0}=10$, the straight wedge without the blunt forebody can initiate the detonation. However, decreasing $M_{0}$ causes the failure of initiation, which can be compensated by increasing the radius $R_{0}$ of the blunt forebody. By adjusting $R_{0}$, two initiation procedures are observed and distinguished: one is the wedge-induced initiation and the other is the blunt forebody-induced initiation. Although both have been independently studied before, in this study, their coexistence is demonstrated, and the mechanism is analyzed for the first time. A theoretical analysis based on the classic initiation theory is performed to elucidate the initiation mechanism, giving a good agreement between the critical radius with numerical results.
\end{abstract}

(c) 2019 Elsevier Masson SAS. All rights reserved.

\section{Introduction}

Detonation waves are supersonic combustion waves spreading in combustible mixtures, in which the leading shock and heat release are closely coupled [1,2]. The detonation engines used in aircrafts receive more and more attention due to their fast heat release and high thermal cycle efficiency. Currently, there are three kinds of detonation engines, namely the pulse detonation engine (PDE) [3], rotating detonation engine (RDE) [4,5] and oblique detonation engine (ODE) [6]. The ODE is considered as an appropriate concept for hypersonic air-breathing propulsion operating at the higher flight Mach number than the Scramjet (supersonic combustion ramjet).

In early research, the oblique detonation wave (ODW) was usually simplified to be the oblique shock wave with a post-shock heat release zone [7]. However, subsequent studies [8-14] demonstrated that an ODW is composed of a non-reactive oblique shock, an induction zone, a set of deflagration waves and an ODW. Recently, there have been several research studies on the surface instabil-

\footnotetext{
* Corresponding author at: State Key Laboratory of High Temperature Gas Dynamics, Institute of Mechanics, Chinese Academy of Sciences, Beijing, 100190, China.

E-mail address: huzm@imech.ac.cn (Z. Hu).
}

ity of ODW [15-20], illustrating and quantifying the formation of fine-scale structures on an oblique detonation surface. For briefness, many past numerical studies [8-20] used simplified chemical reaction models, such as the one-step irreversible Arrhenius kinetic model, which has interior flaws in the initiation study. Recent studies [21-24] have proved that the complex chemistry will considerably influence the detonation characteristics, so ODW research based on a detailed chemical model is necessary. This study mainly uses hydrogen-air mixtures, whose detailed chemical mechanisms have been widely researched in recent years. The ODW structures affected by the inflow Mach numbers and gas-dynamic parameters have been previously simulated and discussed [25]. The inflow inhomogeneity has been previously modeled and simulated to study the ODW produced by fuel injection, demonstrating the reaction surface distortion and morphology variation of the ODW structures [26]. The current study showed that there are two initiation mechanisms of the structures of the wedge-induced ODWs in a hydrogen-air mixture, which are called the kinetics-controlled mechanism and wave-controlled mechanism. The dependence of the initiation length on different parameters, including the wedge angle, flight Mach number, and inflow Mach number has been examined taking the real flight condition into account [27] and the results showed that when the flight Mach number decreases, the initiation length will rapidly increase. 


\section{Nomenclature}

$(x, y) \quad$ physical coordinate system

$(\xi, \eta) \quad$ Cartesian coordinate system

$\mathrm{e}_{\text {inlet }}$ increased energy

$C_{D} \quad$ drag coefficient

$E_{\text {critical critical initiation energy }}$

$F_{D, c r}^{c y l i n d e r}$ drag force work

$e^{D, c r} \quad$ total energy

$h \quad$ enthalpy

$i \quad$ gas species index

$J \quad$ Jacobi matrix determinant

$M_{0} \quad$ flight Mach number

$M_{1} \quad$ inflow Mach number

$n$ species' number

$p \quad$ pressure

$p_{1} \quad$ inflow pressure

$R \quad$ universal gas constant

$R_{0} \quad$ cylinder radius

$\begin{array}{ll}R_{c r} & \text { critical cylinder radius } \\ t & \text { time } \\ T & \text { temperature } \\ u, v & \text { velocity in horizontal and vertical dimensions } \\ U_{0} & \text { flight velocity of the aircraft } \\ U_{1} & \text { inflow velocity } \\ U_{1 x}, U_{1 y} & \text { inflow velocities in the horizontal and vertical direc- } \\ & \text { tions in the aircraft coordinate system } \\ w & \text { molecular weight } \\ \alpha & \text { shape factor } \\ \beta & \text { oblique shock angle } \\ \gamma & \text { specific heat ratio } \\ \rho & \text { density } \\ \rho_{1} & \text { inflow density } \\ \Phi & \text { deflection angle } \\ \omega & \text { mass production rate }\end{array}$

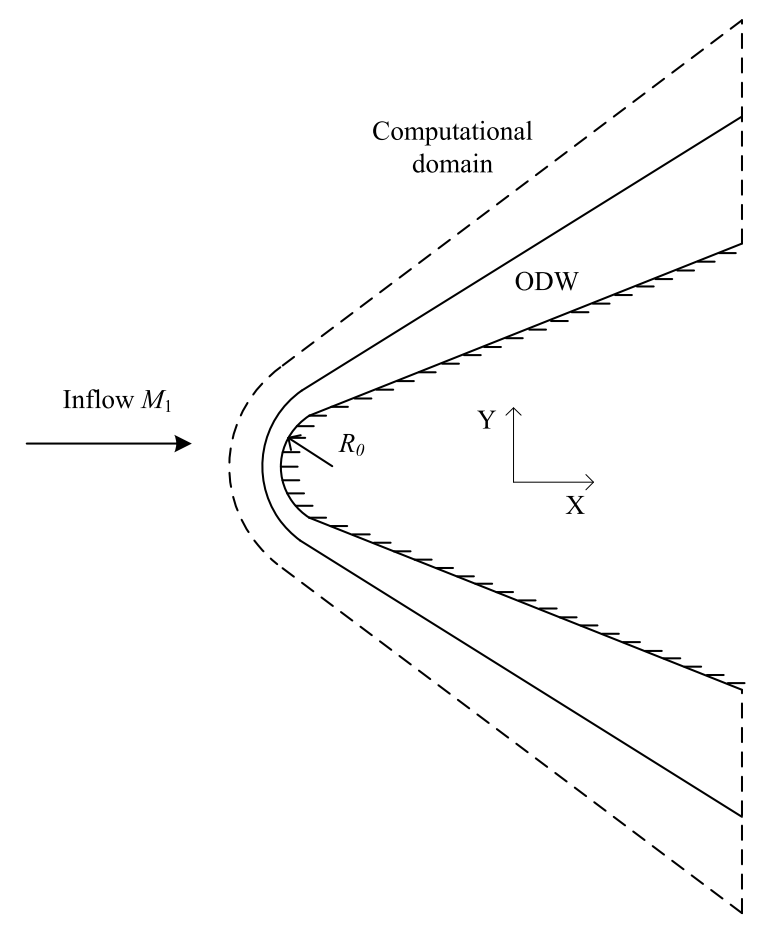

Fig. 1. Sketch of blunt wedge simulation.

In order to induce the ODW in a wide range of flight Mach numbers at high altitudes, the blunt body is set to increase the possibility of initiation. Kaneshige [28] used a blunt body with a ball shape to induce the detonation wave and the experimental results demonstrated that the flow field is different from the ODW flow field induced by a wedge. There are three structures, namely shock with combustion, deflagration to detonation and direct initiation. Vasiljev [29] and Lee [30] studied the influence of the blunt body by theoretical analysis, which is based on the idea that the energy increase is related to the resistance of the blunt body. This study investigated the effect of the blunt body, which is in the front of the wedge, at the flight altitude of $30 \mathrm{~km}$. Vasiljev-Lee's theory is used to analyze the results and predict the critical radius of the blunt body at other flight Mach number and the predicted value agrees with the simulation result.

\section{Physical and mathematical methods}

A schematic of the ODW induced by a wedge with a blunt body in a combustible gas mixture is shown in Fig. 1. For clarity, the blunt body is set as a cylinder. A bow shock forms near the blunt body and if the shock is strong enough, it will induce an ODW upon the wedge. The computational domain is shown in the region enclosed by the dashed lines. Like previous numerical studies on oblique detonations [25], the present analysis is based on the two-dimensional multi-species Euler equations written as follows:

$\frac{\partial \tilde{U}}{\partial t}+\frac{\partial \tilde{F}}{\partial \xi}+\frac{\partial \tilde{G}}{\partial \eta}=\tilde{S}$

where:

$\tilde{U}=\frac{U}{J}, \quad \tilde{F}=\frac{1}{J}\left(\xi_{x} F+\xi_{y} G\right), \quad \tilde{G}=\frac{1}{J}\left(\eta_{x} F+\eta_{y} G\right), \quad \tilde{S}=\frac{S}{J}$

with

$U=\left\{\begin{array}{c}\rho_{1} \\ \vdots \\ \rho_{n} \\ \rho u \\ \rho v \\ e\end{array}\right\}, \quad F=\left\{\begin{array}{c}\rho_{1} u \\ \vdots \\ \rho_{n} u \\ \rho u^{2}+p \\ \rho u v \\ (e+p) u\end{array}\right\}, \quad G=\left\{\begin{array}{c}\rho_{1} v \\ \vdots \\ \rho_{n} v \\ \rho u v \\ \rho v^{2}+p \\ (e+p) v\end{array}\right\}$,

$S=\left\{\begin{array}{c}\omega_{1} \\ \vdots \\ \omega_{n} \\ 0 \\ 0 \\ 0\end{array}\right\}$

and

$\frac{1}{J}=\frac{\partial(\xi, \eta)}{\partial(x, y)}=\left|\begin{array}{cc}\xi_{x} & \xi_{y} \\ \eta_{x} & \eta_{y}\end{array}\right|=\xi_{x} \eta_{y}-\xi_{y} \eta_{x}$

In the above equations, the total density and total energy are calculated by:

$\rho=\sum_{i=1}^{n} \rho_{i}, \quad e=\rho h-p+\frac{1}{2} \rho\left(u^{2}+v^{2}\right)$ 
where the specific enthalpy can be written as $h=\sum_{i=1}^{n} \rho_{i} h_{i} / \rho$ with $h_{i}$ obtained from the thermodynamic data of each individual species. The equation of the state is

$p=\sum_{i=1}^{n} \rho_{i} \frac{R}{w_{i}} T$

where $w_{i}$ is the molecular weight, $T$ is the gas temperature and $\omega_{i}$ is the species' specific mass production rate determined by the chemical reaction model.

The governing equations are discretized on Cartesian grids and solved using the AUSMPW+ scheme [31]. The latest $\mathrm{H}_{2} / \mathrm{O}_{2}$ kinetic model for high-pressure combustion [32-34] is used here, and it involves 27 reversible elementary reactions among the 8 species $\left(\mathrm{H}_{2}, \mathrm{O}_{2}, \mathrm{H}_{2} \mathrm{O}, \mathrm{H}, \mathrm{O}, \mathrm{OH}, \mathrm{HO}_{2}\right.$, and $\left.\mathrm{H}_{2} \mathrm{O}_{2}\right)$ with 1 nonreactive species $\left(\mathrm{N}_{2}\right)$. The thermodynamic properties of the chemical species are evaluated from the 9-coefficient NASA polynomial representation [35]. This code was applied to reproduce the same case as that in a previous study [36] and the same ODW structure was obtained, so it is not shown here. A stoichiometric hydrogen-air mixture with $\mathrm{H}_{2}: \mathrm{O}_{2}: \mathrm{N}_{2}=2: 1: 3.76$ is used. The uniform flow is set as the initial condition, whose velocities are calculated from the inflow Mach number $M_{1}$. The reflecting boundary condition is used on the wedge surface and the wedge angle is $15^{\circ}$. The left boundary, up boundary and down boundary are set as the inflow boundary. The right boundary is interpolated under the assumption of zero first-order derivatives for all flow parameters.

\section{Numerical results and discussion}

\subsection{ODW structures induced by a straight wedge}

To simulate the flow dynamics in an ODE, the flight conditions need to be prescribed to determine the inflow pressure and temperature. An air-breathing aircraft equipped with an ODE is typically designed to operate at high altitude (about 25-35 km) and can cover a range of Mach numbers. Considering a flight altitude of $30 \mathrm{~km}$ and a flight Mach number $M_{0}$ of $8-10$, the ambient flow is pre-compressed twice by weak oblique shock waves in the aircraft inlet. The implicit relation between the oblique shock angle $\beta$ and deflection angle $\Phi$ is given by:

$\tan ^{3} \beta+A \tan ^{2} \beta+B \tan \beta+C=0$

where

$$
\begin{aligned}
A & =\frac{1-M^{2}}{\tan \Phi\left[1+(\gamma-1) M^{2} / 2\right]} \\
B & =\frac{1+(\gamma+1) M^{2} / 2}{1+(\gamma-1) M^{2} / 2} \\
C & =\frac{1}{\tan \Phi\left[1+(\gamma-1) M^{2} / 2\right]}
\end{aligned}
$$

Using the above equations, the oblique shock angle $\beta$ can be calculated, which can then be used to determine the inflow pressure and temperature. Considering a typical deflection angle $\Phi=$ $12.5^{\circ}$, the corresponding static pressure, temperature, inflow Mach number $M_{1}$ and $C-J$ Mach number $M_{C J}$ for each of these cases are listed in Table 1. The inflow Mach number is always greater than C-J Mach number. This means that the oblique detonation waves do not propagate upstream after initiation.

In order to distinguish the effects of the blunt body, the ODWs induced by a half-infinite wedge are first simulated. The flow fields of the ODWs with flight Mach number $M_{0}=10$ are shown in Fig. 2. There is no abrupt change between the oblique shock wave
Table 1

Static pressure, temperature, inflow Mach number $M_{1}$ and C-J Mach number $M_{C J}$ in the cases of different flight Mach numbers $M_{0}$.

\begin{tabular}{lllll}
\hline $\begin{array}{l}\text { Flight Mach } \\
\text { number } M_{0}\end{array}$ & $p(\mathrm{kPa})$ & $T(\mathrm{~K})$ & $\begin{array}{l}\text { Inflow Mach } \\
\text { number } M_{1}\end{array}$ & $\begin{array}{l}\text { C-J Mach } \\
\text { number } M_{C J}\end{array}$ \\
\hline 10 & 56.0 & 1020.6 & 4.3 & 2.5 \\
9 & 44.4 & 891.9 & 4.1 & 2.7 \\
8 & 34.3 & 775.6 & 3.9 & 2.9 \\
\hline
\end{tabular}

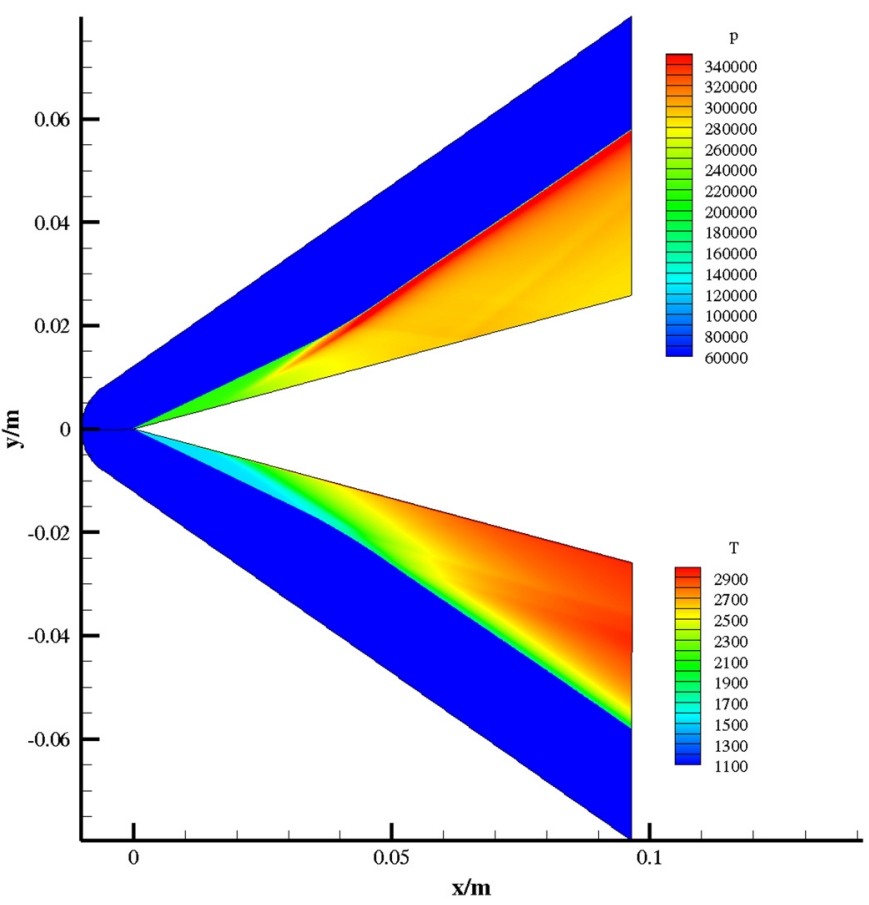

Fig. 2. Pressure and temperature of the ODW structures with $M_{0}=10$. (For interpretation of the colors in the figure(s), the reader is referred to the web version of this article.)

(OSW) face and the ODW face, and the transverse shock is not observed. According to a previous study [36], the initiation process is controlled by kinetics. The entire ODW structure is shown in Fig. 2 and the wedge length is $0.1 \mathrm{~m}$.

The comparison of the results of the resolution tests is shown in Fig. 3, with the results from the high-resolution and low-resolution tests displayed in the upper and lower parts of the image, respectively. In the low-resolution simulation, the grid size was $0.2 \mathrm{~mm}$ and the number of grids was $1000 * 300$, while in the highresolution simulation the number of grids used was $2000 * 600$, doubling the number of grids in both directions and the grid size was $0.1 \mathrm{~mm}$. Apart from some very subtle differences, the results between these two resolutions are almost the same. Therefore, the grid size of $0.2 \mathrm{~mm}$ is adequate to study the ODW structure.

Decreasing $M_{0}$ induces the failure of the initiation process. When the flight Mach number decreases, the initiation zone becomes longer in the case of $M_{0}=9$, as shown in Fig. 4 . And when the flight Mach number is $M_{0}=8$, only OSWs exist in the simulation zone. This result indicates that the ODW flow field is very sensitive to the flight Mach number. If the flight Mach number changes in the range of $8-10$, there will be a large initiation zone or the ODW will not even be induced by the wedge, which should be avoided in the oblique detonation engine. In Fig. 4, there is a slightly high-temperature near-wall zones which is caused by the sharp point. Actually, the line $y=0 \mathrm{~m}$ can be thought as an axisymmetric boundary and the sharp point can be seen as an $R_{0}=0$ blunt body. 


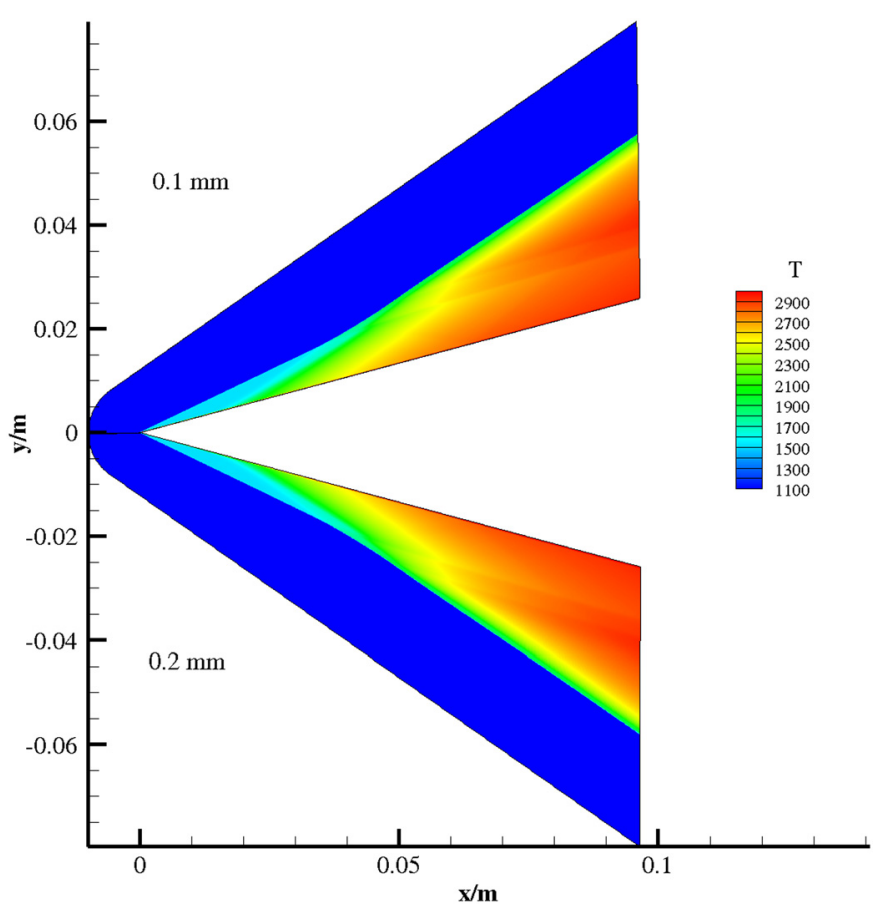

Fig. 3. Temperature of the ODW structures with $M_{0}=10$.

The comparison of calculated and experimental results [37] is shown in Fig. 5. The initial conditions are consistent with the experimental data. The inflow Mach number $M_{1}=6.46$ and the pressure $p=42.7 \mathrm{kPa}$. Here the calculated result is based on axisymmetric two-dimensional multi-species Euler equation and the $y=0$ interface is an axisymmetric boundary. The calculated results show that the combustion and the shock wave are decoupled. This phenomenon was also found in experiments.

\subsection{ODW structures induced by a blunt wedge}

A blunt wedge successfully induces an ODW in the case when $M_{0}=8$ or 9 . For the purpose of inducing an ODW in a wide range of flight Mach number, the blunt body is set at the front of the wedge, namely the blunt wedge. The temperature field of the ODW induced by a blunt wedge whose blunt body is a cylinder with an

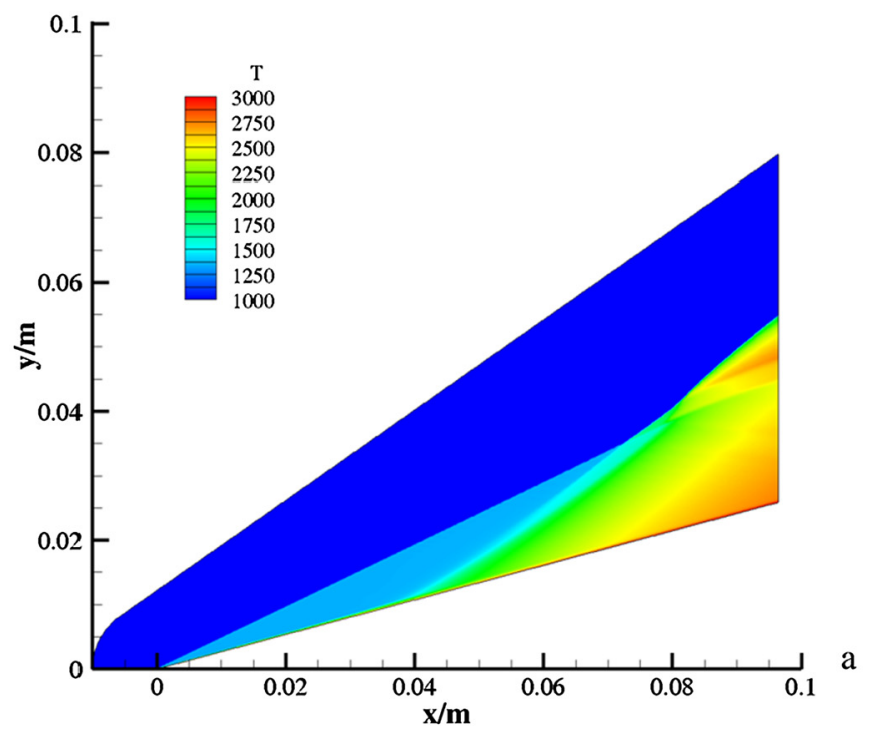

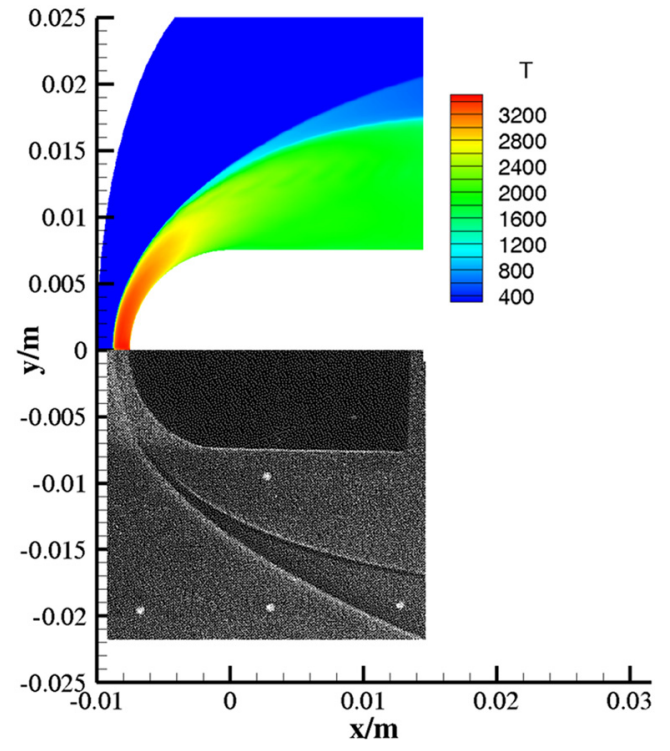

Fig. 5. The comparison of calculated and experimental results.

$R_{0}=5 \mathrm{~mm}$ is shown in Fig. 6 . The temperature increases to about $3,000 \mathrm{~K}$ after the bow shock as shown in Fig. 6 and the ODWs are induced directly by the wedge with the help of the high pressure and temperature zones. Although the temperature declines due to the expansion, the temperature is higher than that in Fig. 4, which demonstrates that the blunt wedge accelerates the chemical reaction, which more easily induces the ODW than a straight wedge. A comparison of the results presented in Fig. 6a and Fig. 6b reveals that the temperature in Fig. $6 a$ is higher because the inflow parameters are different, as can be observed in Table 1 .

The temperature along $y=0.02,0.03$ and $0.04 \mathrm{~m}$ of different grid sizes with $M_{0}=9$ and $R_{0}=5 \mathrm{~mm}$ is shown in Fig. 7. The grid sizes are the same with Fig. 3. Fig. 7 shows that refining the grid size has little effect on the calculation results and the gird size of $0.2 \mathrm{~mm}$ is also adequate to study the ODW structure in blunt wedge.

The pressure and temperature along $y=0,0.01$ and $0.03 \mathrm{~m}$ of the flow fields displayed in Fig. 6b are shown in Fig. 8, illustrating the parameters of three typical positions. Due to the blunt body effect, the pressure and temperature rise very quickly on the

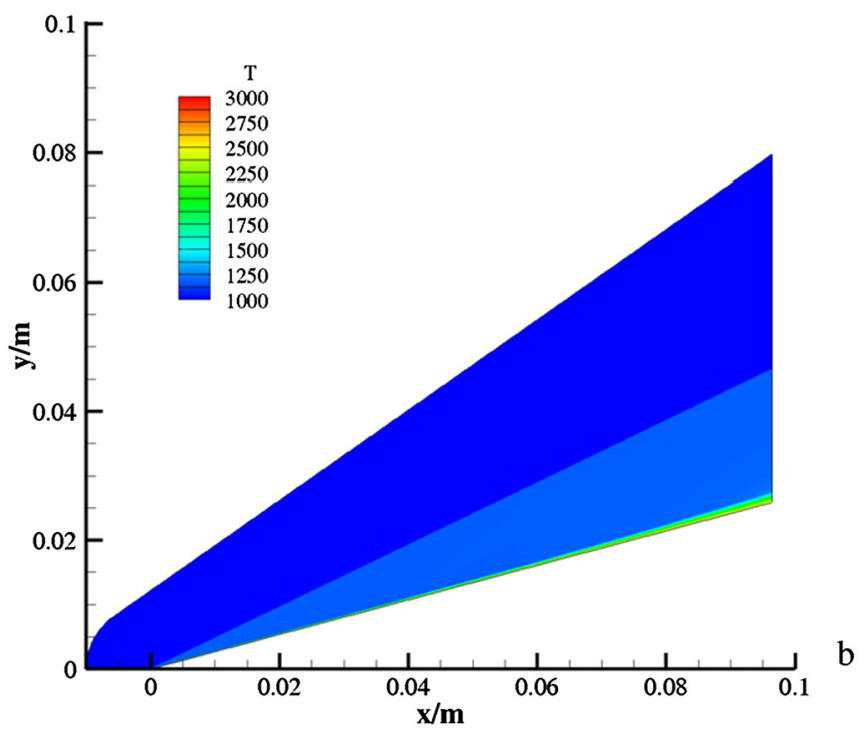

Fig. 4. Temperature of the ODW structures with $M_{0}=9$ (a) and 8 (b). 

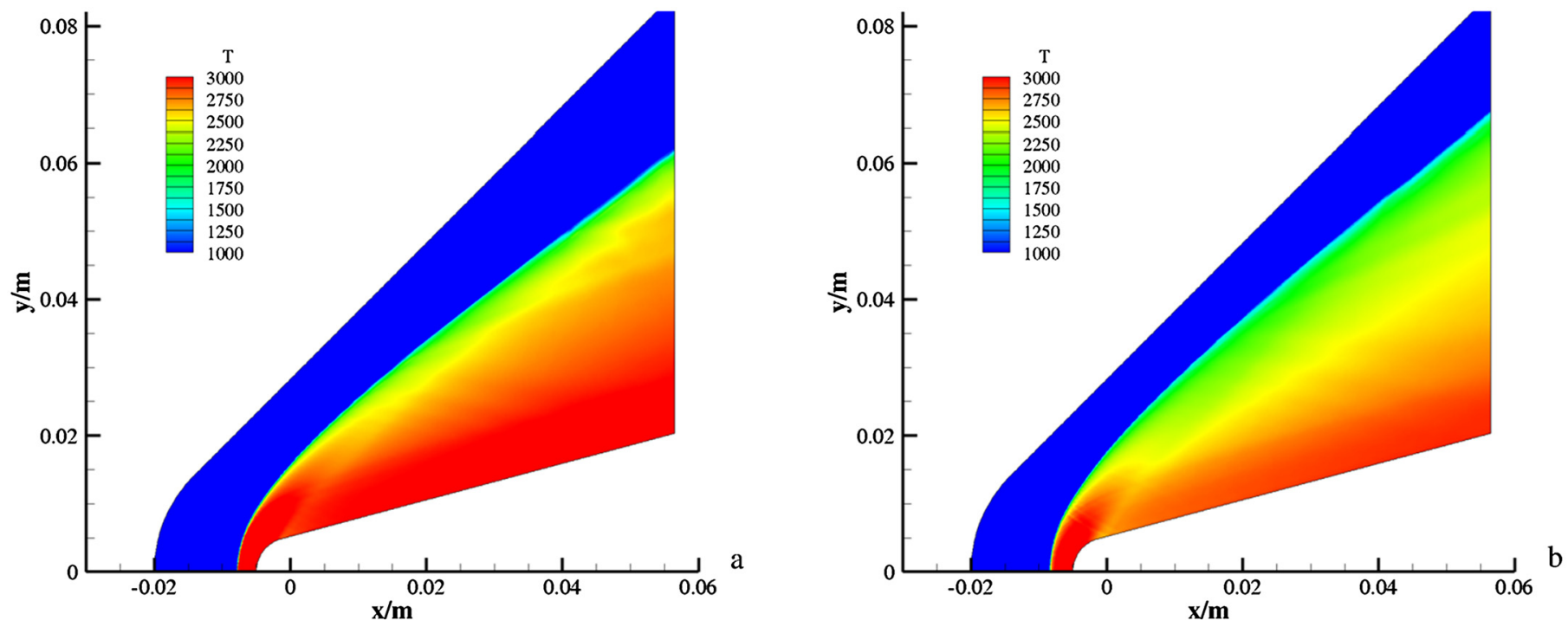

Fig. 6. Temperature of the ODW structures with $M_{0}=9$ (a) and 8 (b), $R_{0}=5 \mathrm{~mm}$.

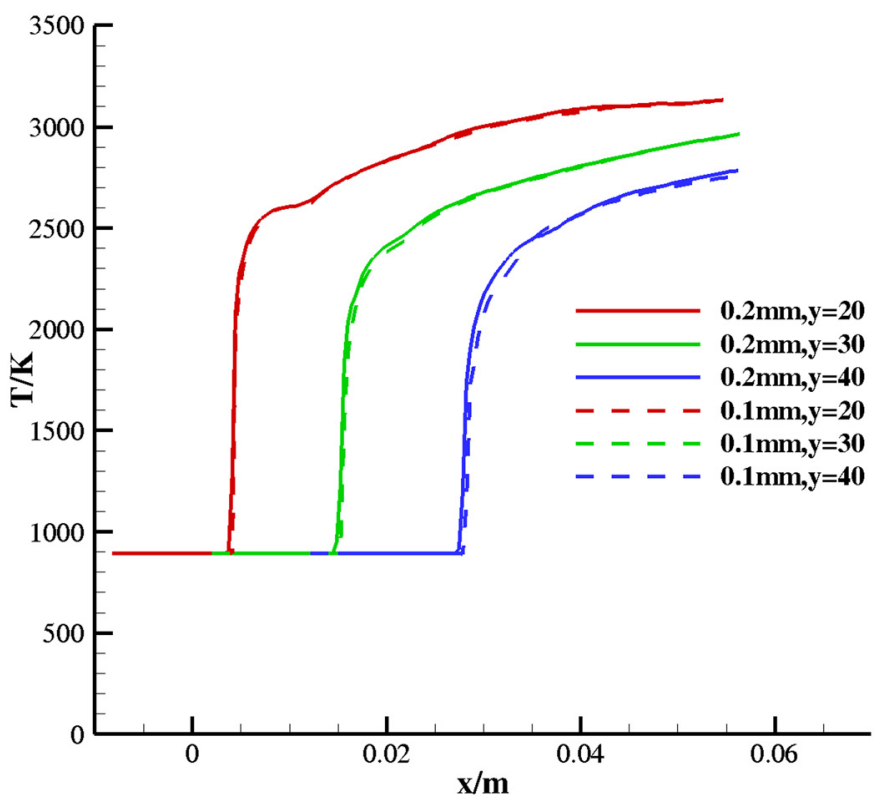

Fig. 7. Temperature of the ODW structures with $M_{0}=9$ along $y=0.02,0.03$ and $0.04 \mathrm{~m}$.

line $y=0 \mathrm{~m}$. The expansion influence can be distinguished along the line $y=0.01 \mathrm{~m}$. The temperature rises after the shock and declines due to the expanding waves. However, the expansion effects are too weak to quench the detonation, and the temperature rises again due to the chemical reaction. When the line is far from the blunt body, e.g., $y=0.03 \mathrm{~m}$, the temperature keeps rising after the shock. In short, the blunt forebody induces an ODW and the expansion influence produces a little difference in the flow field. Accordingly, this ODW structure is referred to as the blunt forebody-induced initiation.

\subsection{Effects of the blunt body radius}

When the radius of the blunt body becomes small, the ODW cannot be directly induced by a blunt wedge and the flow field is different from blunt forebody-induced initiation. As shown in Fig. 9a, the ODW quenches near $x=0.02 \mathrm{~m}$ and starts again near $x=0.04 \mathrm{~m}$ in the case of $M_{0}=9, R_{0}=2 \mathrm{~mm}$. Due to the decoupling of the OSW and chemical reaction, the face of the OSW

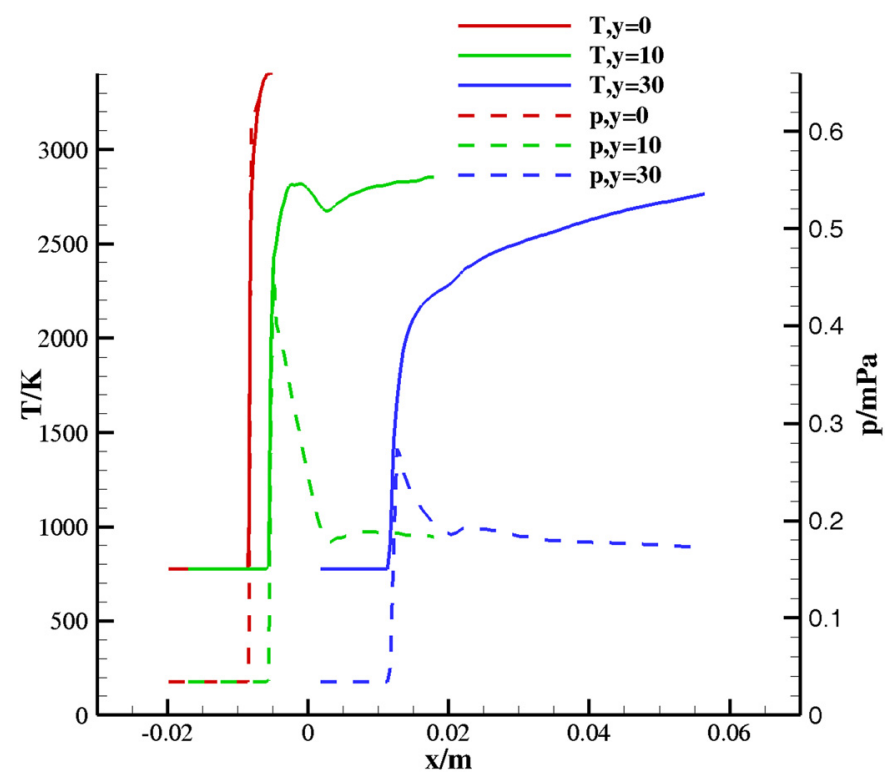

Fig. 8. Pressure and temperature along $y=0,0.01$ and $0.03 \mathrm{~m}$ with $M_{0}=8, R_{0}=$ $5 \mathrm{~mm}$.

becomes concave and its position is very different from that in Fig. 6a. In this situation, the ODW is induced again by the wedge effect so the flow field is referred to as wedge-induced initiation. The pressure and temperature along the line of $y=0.01,0.02$ and $0.03 \mathrm{~m}$ of the flow field displayed in Fig. 9a are shown in Fig. 10a. In the line of $y=0.01$ and $0.03 \mathrm{~m}$, the OSW couples with the release of the chemical heat, while in the line of $y=0.02 \mathrm{~m}$, the release of the chemical heat is separate from the OSW and the highest pressure occurs behind the OSW. In comparison, in the case when $M_{0}=8, R_{0}=2 \mathrm{~mm}$, the chemical reaction decouples from the OSW near $x=0.01 \mathrm{~m}$ and the ODW cannot be reduced again. The changes in pressure and temperature along $y=0.01$, 0.02 and $0.03 \mathrm{~m}$, which show the decoupling of the chemical reaction and OSW, are shown in Fig. 10b. When the radius is equal to $1 \mathrm{~mm}$, which is shown in Fig. 9c, the initiation zone is longer than that obtained by simulation shown in Fig. 9a. A comparison of the results displayed in Fig. 9b and 9d reveals that with smaller radius changes, the decoupling phenomenon is clearer and there is less induction difference between the blunt wedge and the straight 

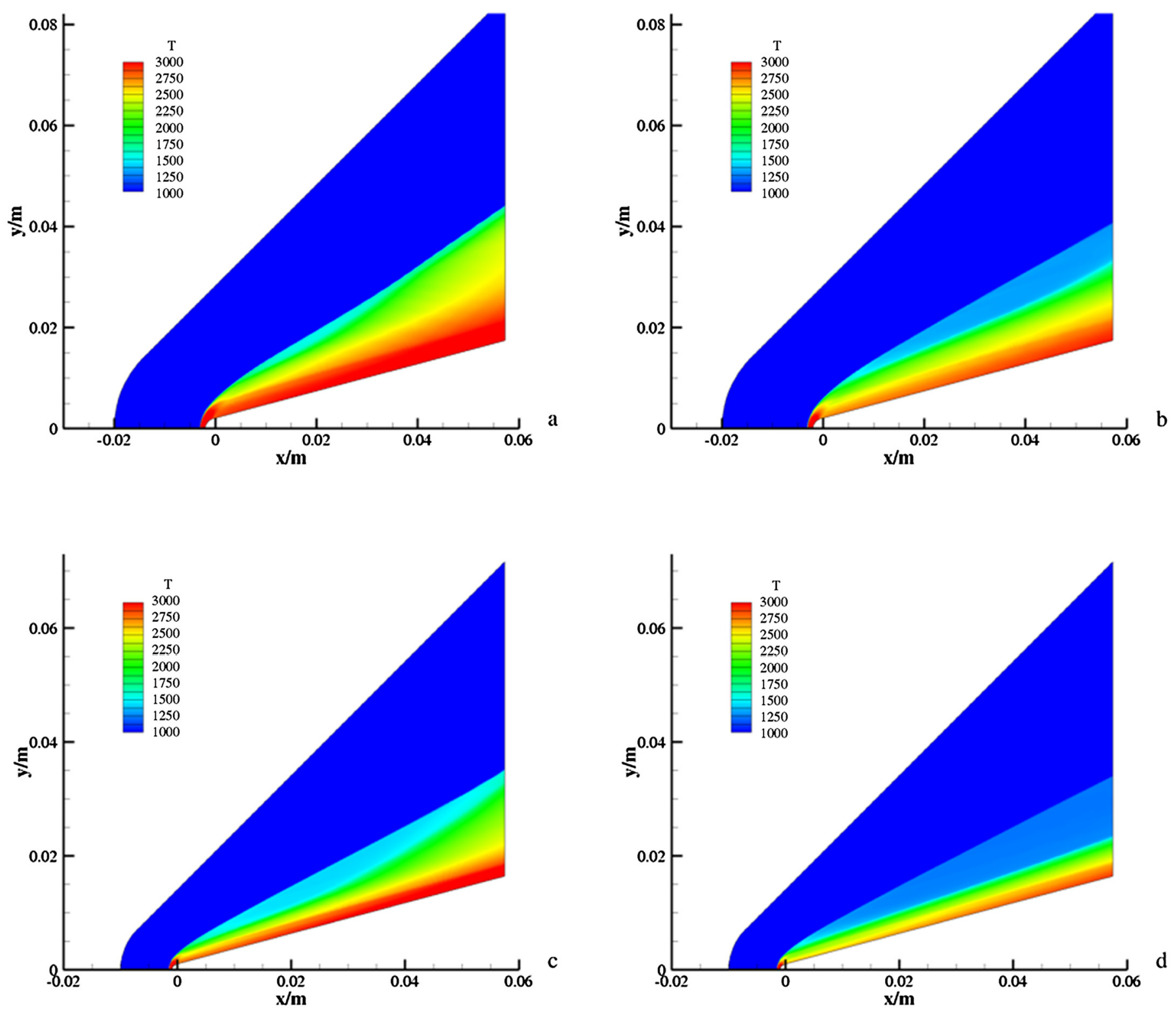

Fig. 9. Temperature of the ODW structures with $M_{0}=9$ (a), 8 (b), $R_{0}=2 \mathrm{~mm}$ and $M_{0}=9$ (c), 8 (d), $R_{0}=1 \mathrm{~mm}$.

wedge. Thus, decreasing the radius changes the initiation mechanism from blunt forebody-induced initiation to the wedge-induced initiation and when the radius is too small, the flow field is similar to that with a straight wedge.

\subsection{Theoretical analysis of the initiation mechanism}

Vasiljev-Lee $[29,30]$ performed some theoretical analysis to determine whether a projectile can induce an ODW. They considered that an ODW will be induced when the drag force work $F_{D, c r}^{c y l i n d e r}$ is larger than the critical initiation energy $E_{\text {critical }}$, i.e.,

$F_{D, c r}^{c y l i n d e r}=E_{\text {critical }}$ tion:

The drag force work can be calculated by the following equa-

$F_{D, c r}^{\text {cylinder }}=\frac{1}{2} \rho_{1} U_{1}^{2} \cdot C_{D} \cdot 2 R_{c r}=\gamma p_{1} M_{1}^{2} \cdot C_{D} \cdot R_{c r}$

Substituting Eq. (10) in Eq. (9) yields

$\gamma p_{1} M_{1}^{2} \cdot C_{D} \cdot R_{c r}=E_{\text {critical }}$ where $\rho_{1}$ is the inflow density, $U_{1}$ is the inflow velocity, $p_{1}$ is the inflow pressure, which are determined by the flight Mach number $M_{0}$ and the deflection angle, $\gamma$ is the specific heat ratio and is equal to $1.4, R_{c r}$ is the critical cylinder radius and $C_{D}$ is the drag coefficient and can be estimated by the modified Newton theory.

$C_{D}=1.84 \alpha$

where $\alpha$ is the shape factor, which is equal to $2 / 3$ for a cylinder.

Then, Eq. (11) can be written as:

$$
1.72 p_{1} M_{1}^{2} \cdot R_{c r}=E_{\text {critical }}
$$

Equation (10) estimates the work done by the drag force of the cylinder. However, in the current study, the inflow gas is pre-compressed by two oblique wedges, which means that these wedges also inject energy to the inflow gas. Considering the compression processes, Eq. (13) can be fixed as:

$\left(1.72 p_{1} M_{1}^{2}+2 \rho_{1} \cdot \mathrm{e}_{\text {inlet }}\right) R_{c r}=E_{\text {critical }}$ 

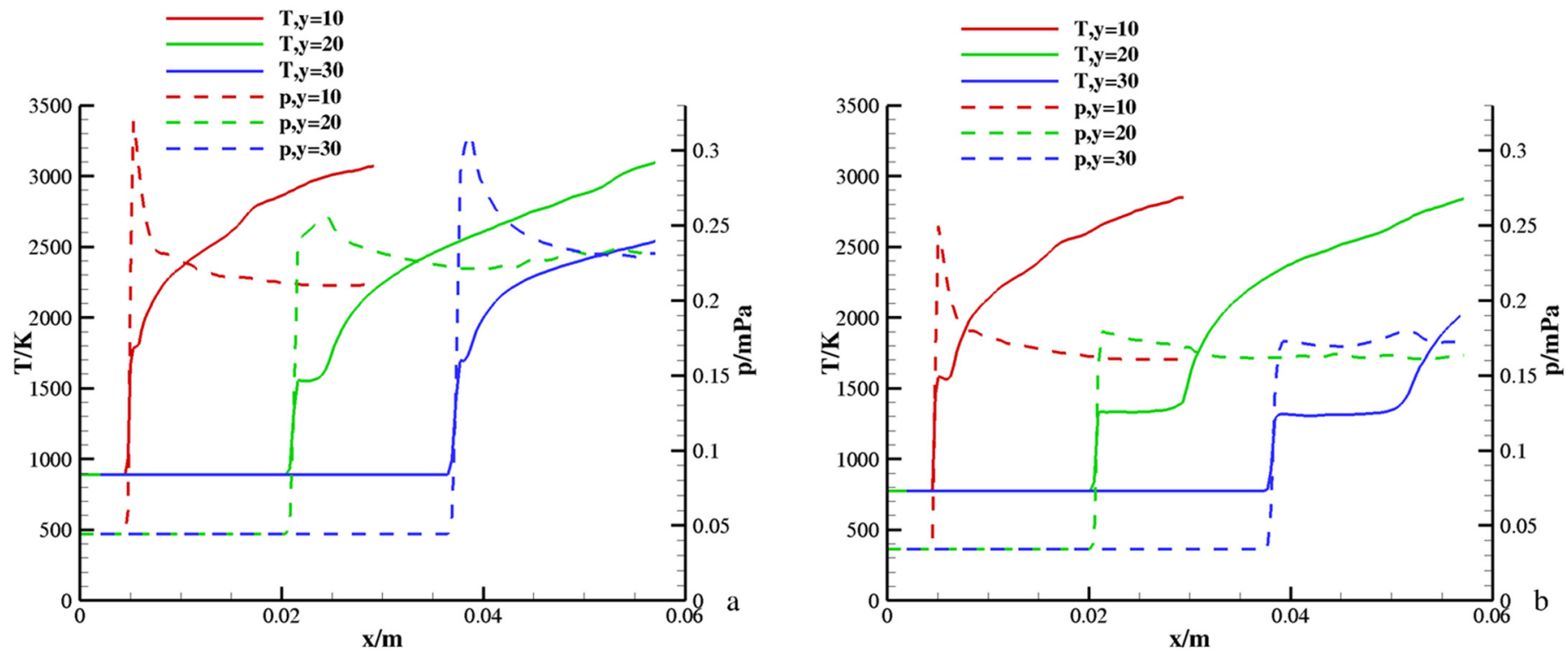

Fig. 10. Pressure and temperature along $y=0.01,0.02$, and $0.03 \mathrm{~m}$ in Fig. 9a (a), and Fig. 9b (b).
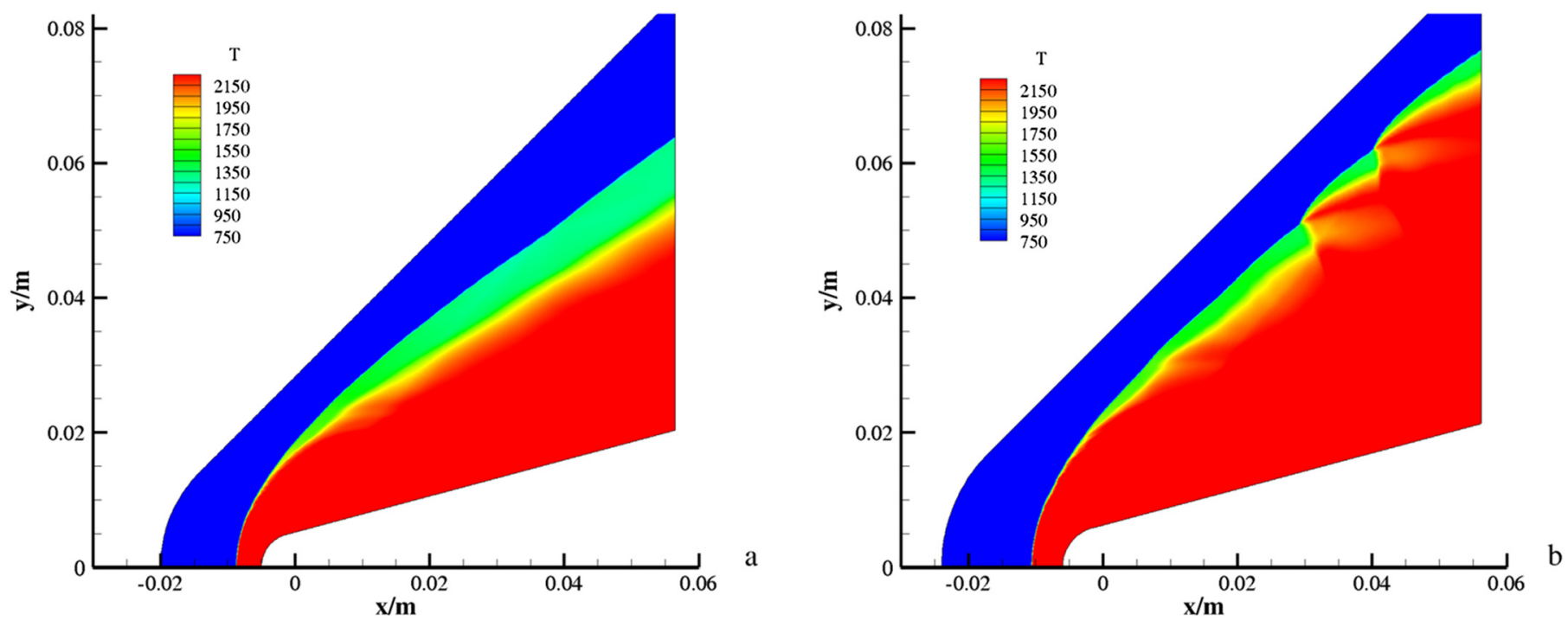

Fig. 11. Temperature of the ODW structures with $M_{0}=7.5, R_{0}=5$ (a) and 6 (b) $\mathrm{mm}$.

where $\mathrm{e}_{\text {inlet }}$ is the increased energy per unit mass, which is determined by the oblique shock wave relationship:

$\mathrm{e}_{\text {inlet }}=h\left(T_{1}\right)-h\left(T_{0}\right)+\frac{1}{2}\left[\left(U_{1 x}-U_{0}\right)^{2}+U_{1 y}^{2}\right]$

where $h\left(T_{1}\right)$ and $h\left(T_{0}\right)$ are the enthalpies in the inflow gas and the flight gas, respectively; $U_{1 x}, U_{1 y}$ are the inflow velocities in the horizontal and vertical directions in the aircraft coordinate system; $U_{0}$ is the flight velocity of the aircraft. Thus, $\frac{1}{2}\left[\left(U_{1 x}-U_{0}\right)^{2}+U_{1 y}^{2}\right]$ is the kinetic energy. Additionally, all the variables in the parentheses of Eq. (14) are dependent on $M_{0}$. Then, the critical radius is determined by the function of the flight Mach number $M_{0}$.

$R_{c r}=\frac{E_{\text {critical }}}{1.72 p_{1} M_{1}^{2}+2 \rho_{1} \cdot \mathrm{e}_{\text {inlet }}}=\frac{E_{\text {critical }}}{f\left(M_{0}\right)}$

The items in Eq. (16) calculated with the flight Mach number $M_{0}=$ 8-10 are summarized in Table 2.

Using different cylinder radius $R_{0}$ and flight Mach number $M_{0}$, the value of $R_{0} \cdot f\left(M_{0}\right)$ can be calculated. If there exist two cylinder radiuses, $R_{1}$ and $R_{2}$, and the detonation wave cannot be in-
Table 2

Different items in Eq. (16).

\begin{tabular}{llllll}
\hline$M_{0}$ & $\begin{array}{l}1.72 p_{1} M_{1}^{2} \\
\left(\mathrm{~J} / \mathrm{m}^{2}\right)\end{array}$ & $\begin{array}{l}2 \rho_{1} \mathrm{e}_{\text {inlet }} \\
\left(\mathrm{J} / \mathrm{m}^{2}\right)\end{array}$ & $\frac{2 \rho_{1} \mathrm{e}_{\text {inlet }}}{1.72 p_{1} M_{1}^{2}} \times 100 \%$ & $\begin{array}{l}f\left(M_{0}\right) \\
\left(\mathrm{J} / \mathrm{m}^{2}\right)\end{array}$ & $\begin{array}{l}R_{c r} \\
(\mathrm{~mm})\end{array}$ \\
\hline 10 & $1.76 \mathrm{E}+06$ & $6.16 \mathrm{E}+05$ & $34.9 \%$ & $2.38 \mathrm{E}+06$ & $2.2-2.7$ \\
9 & $1.29 \mathrm{E}+06$ & $4.60 \mathrm{E}+05$ & $35.8 \%$ & $1.74 \mathrm{E}+06$ & $3.0-3.5$ \\
8 & $8.97 \mathrm{E}+05$ & $3.30 \mathrm{E}+05$ & $37.0 \%$ & $1.22 \mathrm{E}+06$ & $4.3-5.0$ \\
7.5 & $7.30 \mathrm{E}+05$ & $2.75 \mathrm{E}+05$ & $37.7 \%$ & $1.00 \mathrm{E}+06$ & $5.2-6.1$ \\
\hline
\end{tabular}

duced directly by the blunt wedge of $R_{1}$, but it succeeds in being induced directly by the $R_{2}$ blunt wedge, then the critical energy $E_{\text {critical }}$ can be estimated as $R_{1} \cdot f\left(M_{0}\right)<E_{\text {critical }}<R_{2} \cdot f\left(M_{0}\right)$. The simulation results of each Mach number yield one possible range of $E_{\text {critical }}$, and the final range of $E_{\text {critical }}$ can be obtained through their intersection. Using Eq. (16) and the simulation results of $M_{0}=8-10$, the critical energy $E_{\text {critical }}$ is estimated to be between $5.22 \times 10^{3}$ and $6.10 \times 10^{3} \mathrm{~J} / \mathrm{m}^{2}$, and this range of $E_{\text {critical }}$ has the same order of magnitude as that obtained by Lee [30]. Taking the estimated value of $E_{\text {critical }}$ back to Eq. (16) and setting the flight Mach number as $M_{0}=7.5$, the critical radius is predicted to 


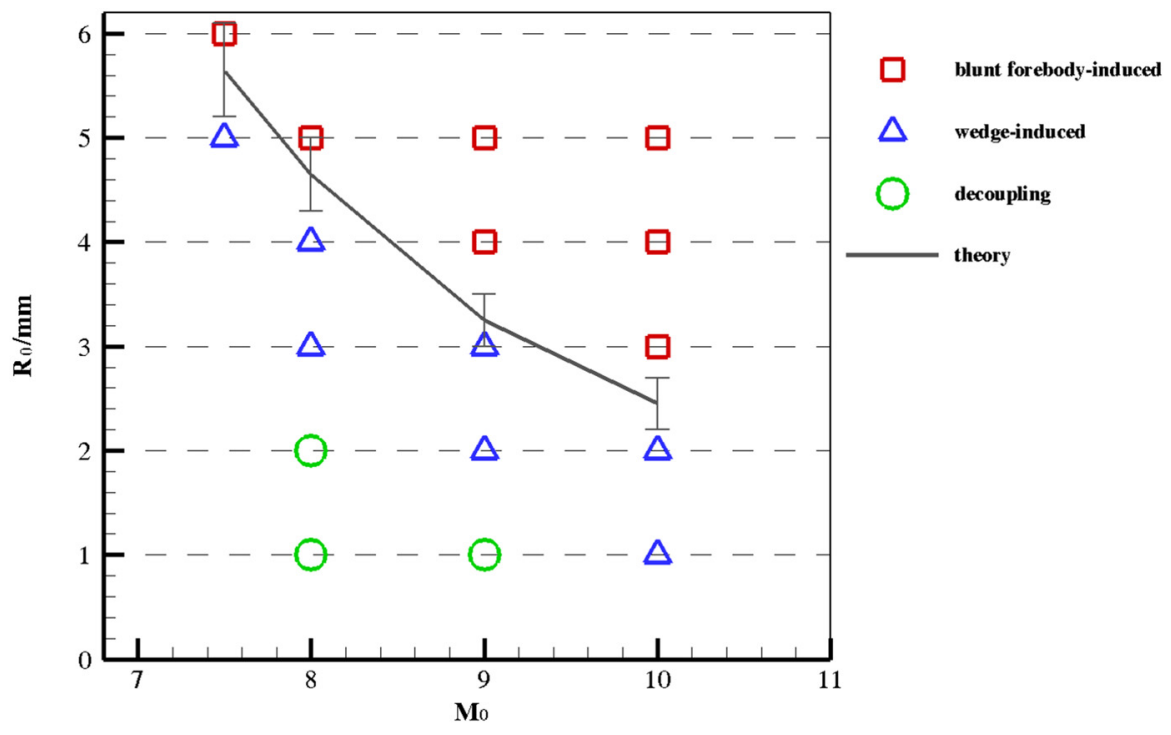

Fig. 12. Simulation ODW structures with different Mach numbers and radiuses and theoretical prediction of critical radiuses.

be between 5.2 and $6.1 \mathrm{~mm}$. To examine the results of the theoretical analysis, two cases of $M_{0}=7.5, R_{0}=5$ and $6 \mathrm{~mm}$ are simulated as shown in Fig. 11. In Fig. 11a, the decoupling of the chemical reaction and OSW can be clearly observed. However, in Fig. 11b, the existence of the ODW cell structures indicates that the blunt wedge successfully induces the ODW. The simulation results and theoretical results, with different flight Mach numbers and radiuses, shown in Fig. 12 revealed that the theoretical predictions agree with the simulation results.

\section{Conclusion}

The initiation of ODWs induced by a blunt wedge is numerically studied using a detailed chemical model. Based on the inflow conditions derived from $M_{0}=8-10$ and an altitude of $30 \mathrm{~km}$, the effects of the different radiuses of the blunt body are simulated. When the radius is large enough, the blunt wedge directly induces an ODW, whereas the wedge induces an ODW with a large region of initiation or may even fail. Two initiation structures are shown in this study: the wedge-induced initiation and the blunt forebodyinduced initiation. These two structures have different shock face shapes, which are influenced by the coupling relationship between the shock face and detonation face. In the case of the directly induced ODW, the work of the drag force is assumed to be larger than the critical initiation energy. Based on the critical initiation energy, a simpler theory is put forward which predicts the critical radius very well in another case.

\section{Declaration of Competing Interest}

There is no competing interest.

\section{Acknowledgement}

The research is supported by the Natural Science Foundation of China (grant numbers 11672308, 11532014).

\section{References}

[1] W. Fickett, W.C. Davis, Detonation: Theory and Experiment, Dover Publications Courier Corporation, 2000

[2] J.H.S. Lee, The Detonation Phenomenon, Cambridge University Press, New York, 2008.

[3] L. Lin, C. Weng, Q. Chen, H. Jiao, Study on the effects of ionization seeds on pulse detonation characteristics, Aerosp. Sci. Technol. 71 (2017) 128-135.
[4] Y. Wang, J. Le, A hollow combustor that intensifies rotating detonation, Aerosp. Sci. Technol. 85 (2019) 113-124.

[5] J. Sun, J. Zhou, S. Liu, Z. Lin, W. Lin, Plume flowfield and propulsive performance analysis of a rotating detonation engine, Aerosp. Sci. Technol. 81 (2018) 383-393.

[6] F.K. Lu, H. Fan, D.R. Wilson, Detonation waves induced by a confined wedge, Aerosp. Sci. Technol. 10 (8) (2006) 679-685.

[7] D.T. Pratt, J.W. Humphrey, D.E. Glenn, Morphology of standing oblique detonation waves, J. Propuls. Power 7 (5) (1991) 837-845.

[8] C. Li, K. Kailasanath, E.S. Oran, Detonation structures behind oblique shocks, Phys. Fluids 4 (1994) 1600-1611.

[9] C. Viguier, L.F. Figueira da Silva, D. Desbordes, B. Deshaies, Onset of oblique detonation waves: comparison between experimental and numerical results for hydrogen-air mixtures, Symp., Int., Combust. 26 (2) (1996) 3023-3031.

[10] L.F. Figueira da Silva, B. Deshaies, Stabilization of an oblique detonation wave by a wedge: a parametric numerical study, Combust. Flame 121 (2000) $152-166$.

[11] H.H. Teng, Z.L. Jiang, On the transition pattern of the oblique detonation structure, J. Fluid Mech. 713 (2012) 659-669.

[12] P. Yang, H. Teng, Z. Jiang, H.D. Ng, Effects of inflow Mach number on oblique detonation initiation with a two-step induction-reaction kinetic model, Combust. Flame 193 (2018) 246-256.

[13] P. Yang, H. Teng, H.D. Ng, Z. Jiang, A numerical study on the instability of oblique detonation waves with a two-step induction-reaction kinetic model, Proc. Combust. Inst. 37 (3) (2019) 3537-3544.

[14] Y. Zhang, P. Yang, H. Teng, H.D. Ng, C. Wen, Transition between different initiation structures of wedge-induced oblique detonations, AIAA J. 56 (10) (2018) 4016-4023

[15] J.Y. Choi, D.W. Kim, I.S. Jeung, F. Ma, V. Yang, Cell-like structure of unstable oblique detonation wave from high-resolution numerical simulation, Proc. Combust. Inst. 31 (2007) 2473-2480.

[16] H.H. Teng, Z.L. Jiang, H.D. Ng, Numerical study on unstable surfaces of oblique detonations, J. Fluid Mech. 744 (2014) 111-128.

[17] M.Y. Gui, B.C. Fan, G. Dong, Periodic oscillation and fine structure of wedgeinduced oblique detonation waves, Acta Mech. Sin. 27 (2011) 922-928.

[18] J. Verreault, A.J. Higgins, R.A. Stowe, Formation of transverse waves in oblique detonations, Proc. Combust. Inst. 34 (2) (2013) 1913-1920.

[19] H. Teng, H.D. Ng, K. Li, C. Luo, Z. Jiang, Evolution of cellular structures on oblique detonation surfaces, Combust. Flame 162 (2015) 470-477.

[20] P. Yang, H.D. Ng, H. Teng, Z. Jiang, Initiation structure of oblique detonation waves behind conical shocks, Phys. Fluids 29 (8) (2017) 086104.

[21] H. Wei, Y. Shang, J. Cai, M. Pan, G. Shu, R. Chen, Numerical study on transition of hydrogen/air flame triggered by auto-ignition under effect of pressure wave in an enclosed space, Int. J. Hydrog. Energy 42 (26) (2017) 16877-16886.

[22] N.N. Smirnov, V.F. Nikitin, L.I. Stamov, D.I. Altoukhov, Supercomputing simulations of detonation of hydrogen-air mixtures, Int. J. Hydrog. Energy 40 (34) (2015) 11059-11074.

[23] B. Zhang, The influence of wall roughness on detonation limits in hydrogenoxygen mixture, Combust. Flame 169 (2016) 333-339.

[24] B. Zhang, X.B. Shen, L. Pang, Y. Gao, Measurement and prediction of detonation cell size in binary fuel blends of methane/hydrogen mixtures, Fuel 172 (2016) 196-199.

[25] H. Teng, Y. Zhang, Z. Jiang, Numerical investigation on the induction zone structure of the oblique detonation waves, Comput. Fluids 95 (2014) 127-131. 
[26] K. Iwata, S. Nakaya, M. Tsue, Wedge-stabilized oblique detonation in an inhomogeneous hydrogen-air mixture, Proc. Combust. Inst. 36 (2) (2017) 2761-2769.

[27] T. Wang, Y. Zhang, H. Teng, Z. Jiang, H.D. Ng, Numerical study of oblique detonation wave initiation in a stoichiometric hydrogen-air mixture, Phys. Fluids 27 (9) (2015) 096101.

[28] M.J. Kaneshige, J.E. Shepherd, Oblique detonation stabilized on a hypervelocity projectile, in: 26th Symp. (Int.) on Combustion, Pittsburgh, 1996, p. 3015.

[29] A.A. Vasiljev, Initiation of gaseous detonation by a high speed body, Shock Waves 3 (4) (1994) 321-326.

[30] J.H.S. Lee, Initiation of detonation by a hypervelocity projectile, in: W.A. Sirignano, A.G. Merzhanov, L. De Luca (Eds.), Advances in Combustion Science: In Honor of Ya. B. Zel'dovich, in: Progress in Astronautics and Aeronautics, vol. 173, AIAA, 1997, pp. 293-310.

[31] K.H. Kim, C. Kim, O.H. Rho, Methods for the accurate computations of hypersonic flows: I. AUSMPW+ scheme, J. Comput. Phys. 174 (1) (2001) 38-80.
32] M.P. Burke, M. Chaos, Y. Ju, F.L. Dryer, S.L. Klippenstein, Comprehensive $\mathrm{H}_{2} / \mathrm{O}_{2}$ kinetic model for high-pressure combustion, Int. J. Chem. Kinet. 44 (7) (2012) 444-474.

[33] J. Li, Z. Zhao, A. Kazakov, F.L. Dryer, An updated comprehensive kinetic model of hydrogen combustion, Int. J. Chem. Kinet. 36 (2004) 566-575.

[34] B.D. Taylor, D.A. Kessle, V.N. Gamezo, E.S. Oran, Numerical simulations of hydrogen detonations with detailed chemical kinetics, Proc. Combust. Inst. 34 (2013) 2009-2016

[35] B.J. McBride, M.J. Zehe, S. Gordon, NASA Glenn Coefficients for Calculating Thermodynamic Properties of Individual Species, 2002.

[36] H. Teng, H.D. Ng, Z. Jiang, Initiation characteristics of wedge-induced oblique detonation waves in a stoichiometric hydrogen-air mixture, Proc. Combust. Inst. 36 (2) (2017) 2735-2742.

[37] H.F. Lehr, Experiments on shock-induced combustion, Astronaut. Acta 17 (1972) 589-597. 